\title{
Advanced Residuals Analysis for Determining the Number of PARAFAC Components in Dissolved Organic Matter
}

The Author(s) 2016

Reprints and permissions: sagepub.co.uk/journalsPermissions.nav DOI: I0.I I77/00037028I5620546 asp.sagepub.com

@SAGE

\author{
Chad W. Cuss', Céline Guéguen ${ }^{2}$, Per Andersson ${ }^{3}$, Don Porcelli ${ }^{4}$, \\ Trofim Maximov ${ }^{5}$, and Liselott Kutscher ${ }^{3}$
}

\begin{abstract}
Parallel factor analysis (PARAFAC) has facilitated an explosion in research connecting the fluorescence properties of dissolved organic matter (DOM) to its functions and biogeochemical cycling in natural and engineered systems. However, the validation of robust PARAFAC models using split-half analysis requires an oft unrealistically large number (hundreds to thousands) of excitation-emission matrices (EEMs), and models with too few components may not adequately describe differences between DOM. This study used self-organizing maps (SOM) and comparing changes in residuals with the effects of adding components to estimate the number of PARAFAC components in DOM from two data sets: MS (I 10 EEMs from nine leaf leachates and headwaters) and LR (64 EEMs from the Lena River). Clustering by SOM demonstrated that peaks clearly persisted in model residuals after validation by split-half analysis. Plotting the changes to residuals was an effective method for visualizing the removal of fluorophore-like fluorescence caused by increasing the number of PARAFAC components. Extracting additional PARAFAC components via residuals analysis increased the proportion of correctly identified size-fractionated leaf leachates from $56.0 \pm 0.8$ to $75.2 \pm 0.9 \%$, and from $51.7 \pm 1.4$ to $92.9 \pm 0.0 \%$ for whole leachates. Model overfitting was assessed by considering the correlations between components, and their distributions amongst samples. Advanced residuals analysis improved the ability of PARAFAC to resolve the variation in DOM fluorescence, and presents an enhanced validation approach for assessing the number of components that can be used to supplement the potentially misleading results of split-half analysis.
\end{abstract}

\section{Keywords}

Parallel factor analysis (PARAFAC), Leaf leachate, Freshwater, Excitation-emission matrix (EEM), Machine learning, Selforganizing map (SOM)

Date received: 12 January 20I5; accepted: 2 July 2015

\section{Introduction}

Dissolved organic matter (DOM) is a complex, heterogeneous, and polymorphous system of interacting molecules that is found in all aquatic environments. DOM has many important functions in natural and engineered systems, such as: providing shelter, energy, and nutrients for microbes; ${ }^{1-3}$ regulating the mobility and toxicity of heavy metals and other pollutants; ${ }^{4-6}$ and generating carcinogenic disinfection by-products during water purification. ${ }^{7-9}$

Fluorescence excitation-emission matrices (EEMs) are produced by aligning fluorescence emission scans measured at several excitation wavelengths. ${ }^{10}$ Parallel factor (PARAFAC) analysis models the variation in fluorescence across a set of EEMs by minimizing the least-squares error in a tri-linear regression of correlated excitationemission (Ex/Em) pairs. ${ }^{11,12}$ The output of the PARAFAC algorithm is thus a set of factors (i.e., components) and corresponding loadings that represent the observed fluorescence patterns in a set of EEMs. Despite uncertainty

\footnotetext{
'Environmental and Life Science Graduate Program, Trent University, Peterborough, ON, Canada

${ }^{2}$ Department of Chemistry, Trent University, Peterborough, ON, Canada ${ }^{3}$ Swedish Museum of Natural History, Department of Geoscience, Stockholm, Sweden

${ }^{4}$ Department of Earth Sciences, University of Oxford, Oxford, UK

${ }^{5}$ International Center for BioGeoScience Educational and Scientific Training (BEST) of North-Eastern Federal University, Yakutsk, Republic of Sakha (Yakutia), Russia
}

Corresponding author:

Céline Guéguen, Department of Chemistry, Trent University,

Peterborough, ON, Canada.

Email: celinegueguen@trentu.ca 
about the molecules and interactions that produce fluorescence in DOM, ${ }^{13,14}$ PARAFAC has been useful for characterizing DOM by end member and tree species, ${ }^{15,16}$ tracing it through ecosystems and ecosystem processes, ${ }^{17,18}$ and assessing its functional effectiveness in natural and engineered systems. $3,6,8,9$

The detection of components in PARAFAC relies upon correlations between Ex/Em pairs, which require consistent relationships through a large portion of a data set. The structure of components modelled by PARAFAC can therefore be confounded by sample sets that are not large enough to describe the variation in fluorescence signatures when the data set is split into parts for split-half analysis. For example, if fluorophores exist in only few samples in a set of EEMs (e.g., leaf leachates ${ }^{16,19}$ ) or if DOM is exposed to treatment along a gradient that causes incremental changes in fluorescence (e.g., size fractionation ${ }^{14,20-22}$ ), then the PARAFAC models generated from split halves will likely contain components with slightly different fluorescence spectra. Such differences may prevent validation using split-half analysis, the success of which in turn requires the exclusion of samples with distinct spectra as "outliers" for the sake of building a coherent model. ${ }^{12}$ Further, a PARAFAC model of these EEMs that is successfully validated using split-half analysis is likely to account for this inconsistent variation by incorporating several constituents into a single component. This is particularly likely for humic-like components, since there is evidence that their fluorescence signature arises from chargetransfer interactions, which can cause the same fluorophore to manifest a slightly different fluorescence signature when the identity of proximate molecules is altered by, for example, conformational changes. ${ }^{13,14}$ Such under-specification of PARAFAC models can lead to inconsistencies in the behavior of components both within and across experiments. The combination of correlation-dependent factor identity and acceptance based on split-half analysis thus places severe limitations on the differences that can be detected between EEMs of DOM with disparate fluorescence properties, and those collected over experimentally controlled gradients, potentially leading to erroneous conclusions about the behavior and identity of fluorescent components. While another more developed PARAFAC model could be applied to EEMs from new DOM samples, it has been found that such application is less sensitive to differences in fluorescence signatures than is a newly developed PARAFAC model. ${ }^{23}$ Further, such application has produced several residual components that were not welldescribed using the pre-existing model. ${ }^{19}$

The limits of split-half analysis for validating the number of PARAFAC components in DOM have been previously recognized, wherein it was noted that the analysis of residuals plays a key role in validation. ${ }^{11,12}$ The assessment of patterns in residuals is a vital step for determining the quality of all linear models, and has also been suggested for use with PARAFAC. ${ }^{24}$ However, no rigorous method of residuals analysis exists for DOM researchers. The current study fills this gap by using self-organizing maps ${ }^{25}$ (SOM) and the changes in residuals between PARAFAC models to assess patterns in model residuals, allowing a more refined estimation of the number of PARAFAC components. Machine learning methods are applied to model outputs to compare the amount of relevant information provided by extending the number of components beyond that validated using split-half analysis.

\section{Materials and Methods}

\section{Data Sets and Instrumental Analysis}

Two sets of EEMs with disparate properties were used to illustrate a range of modeling conditions. Detailed data characteristics are shown in Table I. The multisource (MS) data set consisted of IIO distinct EEMs collected from nine different tree species and headwaters, including: six leaf leachates (overwintered silver maple, senescent red maple, senescent reed canarygrass, senescent silver maple, senescent tamarack/larch, and senescent white spruce) and three freshwaters collected during spring freshet (coniferous-dominated, deciduous-dominated, and wetland).

Table I. Characteristics of data sets and resulting PARAFAC models validated by sixfold " $\mathrm{S}_{4} \mathrm{C}_{6} \mathrm{~T}_{3}$ " split-half analysis.

\begin{tabular}{|c|c|c|}
\hline \multirow[b]{2}{*}{ Characteristic } & \multicolumn{2}{|l|}{ Data set properties } \\
\hline & Multisource (MS) & Lena River (LR) \\
\hline Description & Six leaf leachates and three headwaters & Lena river and tributaries \\
\hline EEMs (n) & 110 & 64 \\
\hline Known sources of variation & $\begin{array}{l}\text { 81 EEMs of nine different sources measured at } \\
8-10 \text { different size fractions and } 29 \text { whole samples }\end{array}$ & Whole samples only \\
\hline Duplication & Two-four distinct sub-samples for full EEMs & \\
\hline Outliers removed & 0 & 2 \\
\hline Validated components ( $n$ ) & 3 & 3 \\
\hline
\end{tabular}


Samples were size-fractionated using online asymmetrical flow field-flow fractionation coupled to UV-Vis diode array and fluorescence detectors. ${ }^{26}$ The methods used to produce and fractionate the leachates are detailed in earlier studies, wherein it was found that the optical properties of both size fractions and leaf species were markedly different. $^{16,20,21}$ EEMs of whole samples were also measured.

The Lena River (LR) data set consisted of 64 whole DOM samples collected along the Lena River (Russia) and its tributaries shortly after spring melt in $201 \mathrm{I}$ and 2012 . Samples were filtered on site (pre-combusted $0.7 \mu \mathrm{m}$ GFF) before being sealed in acid-washed, $250 \mathrm{~mL}$ HDPE containers, wrapped with Parafilm, and shipped to Canada for measurement. Samples were not kept cool during shipping, and I-2 months elapsed between sample collection and measurement; hence, microbial processing likely depleted sample-specific signatures. It is expected that the relative variation in optical properties is lower for the potentially degraded, riverine DOM (LR data set) compared to the mixture of fresh leaf leachates and headwaters measured at several size fractions (MS data set).

All samples were diluted below $0.01 \mathrm{AU}$ prior to measuring fluorescence to minimize inner-filtering effects. ${ }^{27}$ All EEMs were measured over Ex/Em wavelength ranges of 200-450/280-600 nm in increments of $1 \mathrm{~nm} / 5 \mathrm{~nm}$ using an Agilent II00 series fluorescence detector. Wavelengths were calibrated following the manufacturer-recommended procedure, and EEMs were measured in ratio (S/R) mode. Blank EEMs of carrier fluid (for size fractions) and ultrapure Milli-Q water (MQW; for whole DOM samples) were measured on each day of analysis, and subtracted from sample EEMs. The area under the Raman water peak of blank EEMs at $E x=350 \mathrm{~nm}$ was used to standardize fluorescence intensity to Raman units. ${ }^{28}$

\section{Preprocessing and PARAFAC Model Parameters}

PARAFAC was applied in Matlab R2010a using the drEEM package. ${ }^{12}$ Noise was trimmed from the edges of all EEMs by removing Ex wavelengths $<230 \mathrm{~nm}$ and $>430 \mathrm{~nm}$, and all Em wavelengths $>550 \mathrm{~nm}$. First- and second-order scatter was removed without interpolation. EEM intensities were normalized to unit norm. ${ }^{12}$

Two outliers were removed from the LR data set due to aberrant fluorescence, but no outliers were removed from the MS data set despite clusters with high leverage. The high-leverage clusters were associated with samples that had very high and low loadings on some components, and corresponded with DOM leached from different tree species, and measurements at the extremes of molecular weight. This affirmed the assumption of higher DOM heterogeneity in the MS data set.

The MS and LR data sets were modeled with non-negativity constraints for $n=3-1 \mathrm{l}$ components. The convergence criterion for all models was set to $10^{-8}$, and all models converged. ${ }^{12}$ To ensure that a robust model was chosen, models of both data sets were produced 10 times from random starting conditions for each value of $n$, and the one with the lowest sum of squares error (SSE) was used. $^{11,12}$ Despite minor fluctuations, the SSE was similar for all 10 models. Models were validated using sixfold splithalf analysis $\left(\mathrm{S}_{4} \mathrm{C}_{6} \mathrm{~T}_{3}\right){ }^{12}$ The change in percentage of total variation explained by adding a component to a model was calculated by subtracting amount explained using $n$ components from that explained using $n+l$ components, and plotted against the number of components in a scree plot. ${ }^{24}$

\section{Self-Organizing Maps and Machine Learning}

The overall average residuals for each PARAFAC model and the residuals averaged by tree species or headwater (MS data set only) were calculated by altering the EvalModel command to output and average the model residuals. "I Changes in the residuals were calculated by subtracting the residuals of models with $n+I$ components from those with $\mathrm{n}$ components.

Kohonen's self-organizing maps ${ }^{25}$ (SOM) were used to detect patterns in the residuals of PARAFAC models. SOM are dual layer (input and output) artificial neural networks that were originally developed for pattern recognition in artificial intelligence research. SOM have been applied to the residuals of linear models for predicting faults in aircraft engines, ${ }^{29}$ and for pattern detection in EEMs, ${ }^{21,30-34}$ aquatic systems, ${ }^{35,36}$ and ecology-toxicology. ${ }^{37,38}$ SOM were applied both to the residuals and factor loadings (i.e., Fmax) from PARAFAC models in order to assess: (I) patterns in the residuals and their relationship with DOM from different tree species; (2) the relative differentiability of DOM by tree species/headwater; and (3) model overfitting by comparing the distributions of component loadings. Residuals and loadings were both scaled to constant variance, and each EEM of residuals was standardized on the interval $(0, I)$ to focus on remaining fluorescence peaks. The methods used to determine map size, initialize the model vectors of map neurons, and ensure optimal fitting are discussed in the supplementary information.

To assess changes in the amount of useful information provided by different PARAFAC models of the MS data set, PARAFAC-modeled samples were classified by species/ headwater by applying the k-nearest neighbors algorithm in Weka (v.3.6.ll; University of Waikato, NZ). Each PARAFAC loading was transformed into the proportion of total fluorescence by dividing its Fmax value by the sum of all Fmax values within its respective EEM. Fluorescence compositions were transformed to the real number line using the centered log-ratio transformation. ${ }^{39}$ Parameter settings for the $k$-nearest neighbor algorithm are discussed in the supplementary information. 


\section{Results and Discussion}

\section{PARAFAC Models, Component Spectra, and Scree Plot}

For both the MS and LR data sets, only three PARAFAC components could be validated by split-half analysis explaining $88.9 \%$ and $98.6 \%$ of total variation, respectively. Sevencomponent models were validated in one of three splits for both data sets; however, the spectra of two components were very similar for the seven-component model of the LR data set, suggesting potential overfitting. ${ }^{12}$ In the MS data set, six samples measured at the highest molecular weights for three tree species (senescent red and sugar maple, white spruce, and reed canarygrass) had loadings of zero on all components in the three and four component models. The lack of loadings for all components suggests that the fluorescence of DOM was below the detection limit, so that variation in fluorescence was primarily attributed to noise. Five samples had loadings of all zeros in the PARAFAC models with five to 12 components. Since the noise in these EEMs was not modeled as any component by the PARAFAC algorithm, it is unlikely that the modeled PARAFAC components contained worrisome levels of noise.

The spectra of most PARAFAC components were typical of those reported for DOM, including multiple proteinpolyphenol-like, fulvic-like, and humic-like varieties (Figs. I and SI-6, SI0-13). However, the LR data set models with eight to II components contained one recurring component with two excitation maxima at 235 and $260 \mathrm{~nm}$, with two corresponding Em maxima at 350 and $430 \mathrm{~nm}$ (Fig. SI5). The eight to II component models of the LR data set were therefore discarded, since chemically meaningful peaks do not exhibit dual emission maxima. ${ }^{12,27}$ The II component model of the MS data set was also discarded, owing to a similar component with one excitation maximum $(255 \mathrm{~nm})$ and two or three emission maxima (approximately 310, 335, and $380 \mathrm{~nm}$; Fig. S9).

In models of the MS data set with six or more components, the spectra of some components were slightly distorted (e.g., departure from sphericity, wrinkled edges), which is usually considered as a sign that noise has been
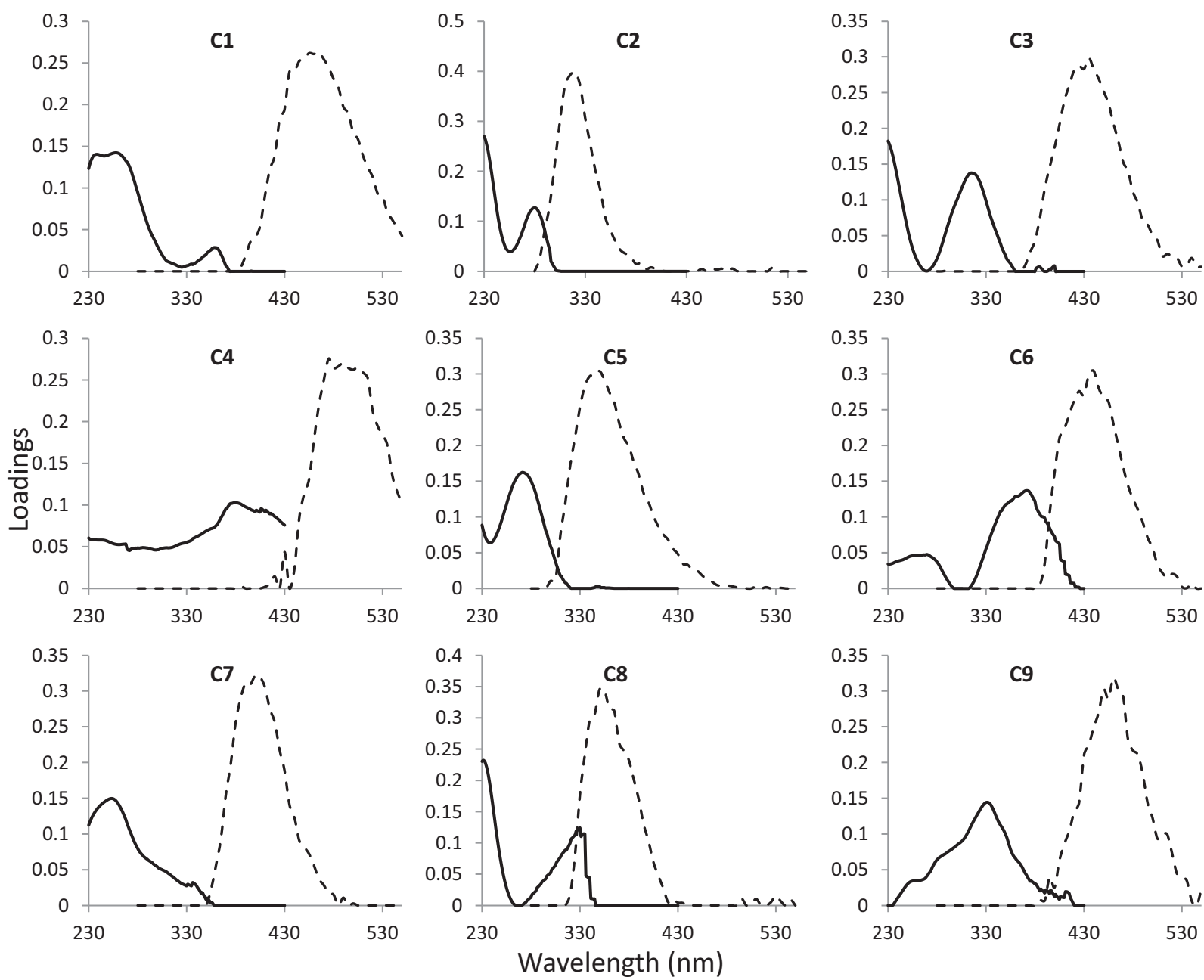

Figure I. Excitation (-) and emission (--) spectra for nine-component PARAFAC model of MS data set. 
included (Figs. S4-S8). Noise is generally undesirable; however, the nature of the phenomena being described by changes in fluorescence throughout the samples should be considered before such components are discarded. In this case the DOM samples were collected along gradients of concentration (i.e., full and size-fractionated EEMs), sizes (range, $0.3 \mathrm{I}-28 \mathrm{kDa}$ ), and tree species/headwaters, all of which demonstrate shifts in the location of peak maxima that may be associated with changing electronic interactions, particularly in regions associated with humic-like material. ${ }^{14,16,21}$ It is therefore reasonable to expect that peak locations may shift slightly from sample to sample, causing distorted edges in the associated component.

If many samples do not contain a particular peak, then the inclusion of some noise is acceptable as model dimensionality increases. This type of noise is expected because the component is modeled using correlations found between Ex/Em pairs through all EEMs, and will therefore likely incorporate noise from correlations in EEMs for which the underlying fluorescent group (or "fluorophore") is absent, minimally expressed, or fluoresces in a slightly different position. For example, if II EEMs from the same tree species in the MS data set (I IO EEMs total) contain a unique fluorophore, then the associated region of fluorescence will contain only noise or the spectra of components that overlap the region for $90 \%$ of the EEMs. Hence, a component defined to explain the variation in Ex/Em pairs across all of the EEMs will contain substantial noise, and consequently may not be smooth and spherical. This is especially relevant for size fractions associated with higher molecular weights, since many of these were measured at low concentrations where the signal-to-noise ratio is lower. Further, higher molecular weight fractions tend to contain a larger proportion of humic-like material in leaf leachates, ${ }^{20,21}$ so that the Ex/Em ranges of the underlying fluorescent groups (or fluorophores) is likely inconsistent across samples due to slight shifts caused by different structures combined with pervasive charge-transfer interactions. ${ }^{14}$ Some evidence of noise should therefore be tolerated in components, especially those that explain fluorescence in the humic-like region $(\mathrm{Em}>400 \mathrm{~nm})$.

If newly added components are primarily noise, then the amount of variance explained will approach a constant value (i.e., the noise level) as the number of components is increased. Although it does not provide a conclusive answer as to the correct number of components, this phenomenon can be detected by plotting the percentage of total variation explained by each component (i.e., a "scree plot"). ${ }^{24} \mathrm{~A}$ "flattening out" of the scree plot typical of noise was evident in the 7-IIC and 8-I IC models of the MS and LR data set, respectively (Fig. SI6). The smooth scree plot for the LR data set suggests that six to seven components are adequate for explaining the observed variation. Given that the scree plot for the MS data set begins to level off at six to seven components, it may be prudent to stop at this level; however, the large amount of known variation and distorted shape of the scree plot (especially for five and nine components) suggests that eight to nine components should also be investigated.

Overfitted PARAFAC models may also contain redundant, highly-correlated components, which can be assessed using the core consistency diagnostic. ${ }^{40,41}$ Although it is suspected that the core consistency diagnostic may under-fit PARAFAC models of DOM, ${ }^{12}$ it may be useful for detecting exceptional correlations between components. In particular, the core consistency diagnostic should become "seriously negative" if highly correlated components are present. ${ }^{42}$ In this study the core consistency decreased steadily with increasing numbers of components, and oscillated around zero for the 10 to II component and nine to II component models in the MS and LR data sets, respectively, giving no clear indication for the point at which overfitting occurs (data not shown).

\section{Visual Inspection of Residuals}

In the overall mean residuals of the MS dataset, residual fluorescence was evident in regions corresponding to humic-, fulvic-, and protein-like peaks, but peak shape was poorly defined (Fig. 2a). On the other hand, the spectra of peaks in the residuals were well defined in plots of the corresponding changes to overall residuals or $\Delta R$ plots (Fig. 2b). The $\Delta R$ plots illustrated the fluorescence that was removed (positive values) and restored (negative values) from the residuals by the transition to models with more components (e.g., $\Delta R_{3-4 C}=$ average residuals from three-component PARAFAC model-average residuals from the four-component PARAFAC model). Peak shapes were easier to discern in $\Delta R$ plots because they were not smoothed by including noise/values near zero from EEMs that did not contain residual fluorescence at these $\mathrm{Ex} / \mathrm{Em}$ pairs (i.e., $\Delta \mathrm{R} \approx 0$ for EEMs in which adding a component had a negligible impact).

The $\Delta R$ plots also displayed the impact of adding a PARAFAC component, which is not always clear from the spectra of the components. For example, the major visual differences between components in the three- and fourcomponent model involved adding a component with maximum $\mathrm{Ex} / \mathrm{Em}=325 / 445 \mathrm{~nm}$ and decreasing the loading on one $(E x / E m=320 / 4 / 0)$ of two peaks in another component, resulting in very different values for the variance explained by two-thirds of the original components (Figs. SI, S2). This resulted in adjustments to three distinct peaks in the in the plot of $\Delta R_{3-4 C}$, with Ex/Em maxima near 260/400, 260/500, and 320/450 nm (Fig. 2b, upper panel), which corresponded with the poorly delineated fluorescence evident in the mean residuals (Fig. 2a, upper panel). Thus, $\Delta R$ plots allow judgements to be made about the spectral shape of the fluorescence modeled by an added component, providing another means by which to judge 
(a)
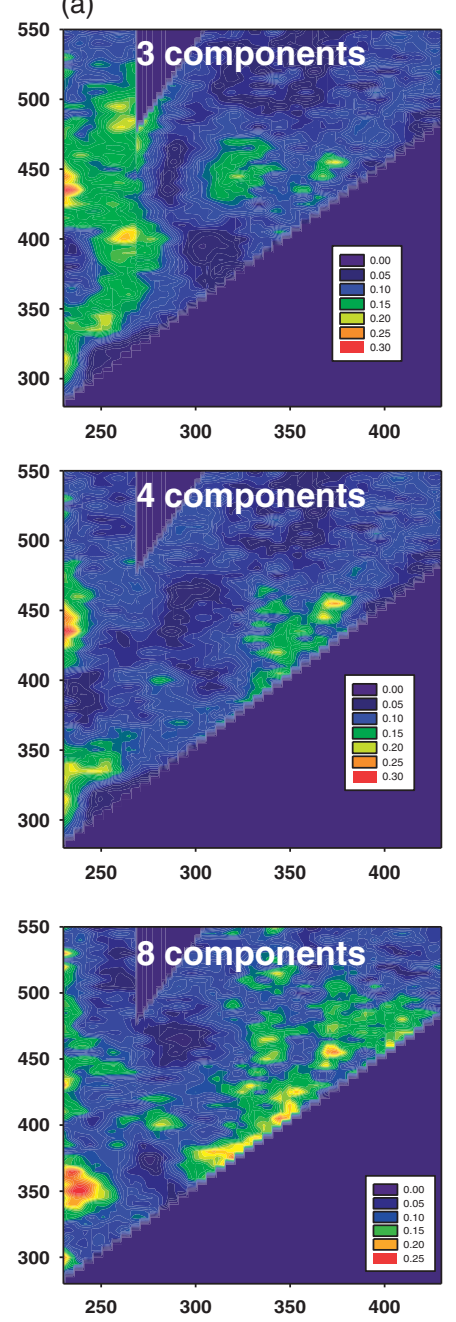

(b)
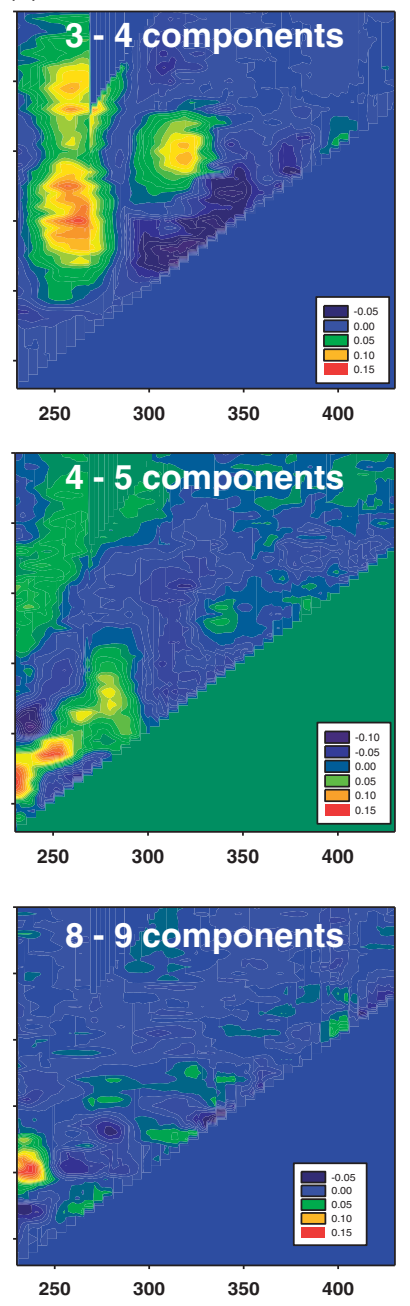

(c)
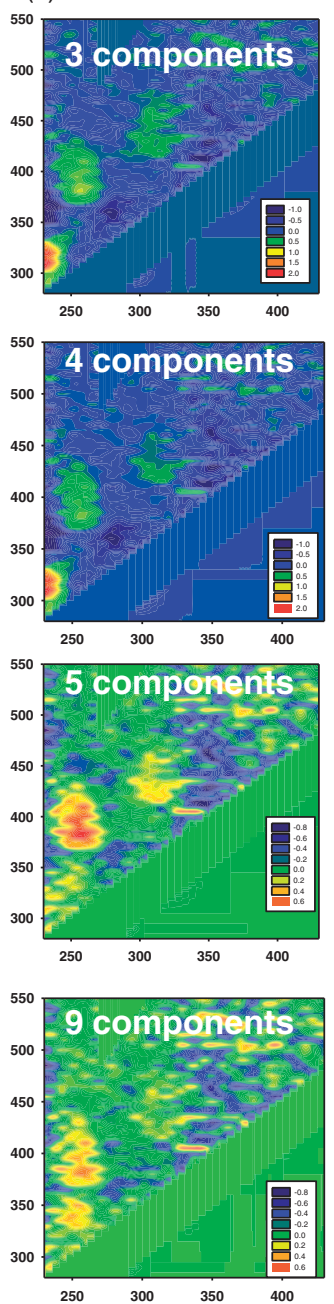

(d)
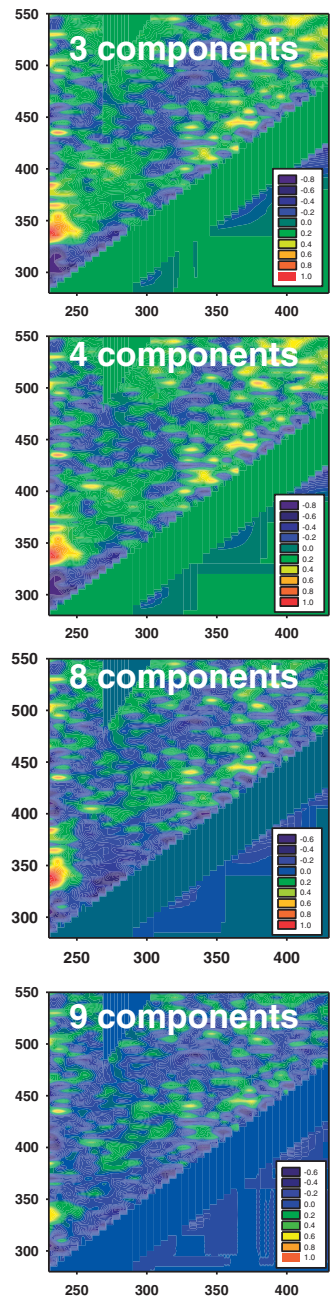

Figure 2. Residuals from selected PARAFAC models of the MS data set shown by (a) overall mean residuals, (b) changes in overall mean residuals (i.e., $\Delta R$ plots), and averaged by tree species for (c) tamarack, and (d) overwintered silver maple. Excitation and emission wavelengths are shown along the $x$ - and $y$-axes, respectively.

model appropriateness: if adding a component results in adjustments to chemically meaningful fluorescence (i.e., not scattered "noise"), and removes peaks that are evident in residuals, then the major features of $\Delta R$ plots should resemble one or more symmetric peak.

It is recommended that the residuals of each DOM sample are viewed to assess the existence of persistent patterns during refinement of the PARAFAC model. ${ }^{1,12}$ However, it can be difficult to see systematic evidence of fluorophores remaining in the residuals when the plots are viewed a few at a time, particularly when the number of EEMs is large and the affected samples are not grouped together. On the other hand, $\Delta \mathrm{R}$ plots enable the visualization of changes to residuals that are caused by adding a component to the PARAFAC model. When peak-like spectral features are the dominant source of variation in residuals, changes to the residuals caused by adding a component (e.g., 3C model-4C model) should be primarily positive (denoting the removal of additional fluorescence rather than simply shifting peak locations) and peak-like (i.e., symmetric, non-random). This was highlighted by the variable structure of the MS data set, because of the species-specific nature of fluorescence in the DOM of leaf leachates. ${ }^{16}$ For example, the protein/polyphenol-like peak remaining in the mean residuals of Tamarack leachates for the four-component PARAFAC model (Fig. 2c) was not readily apparent in the overall mean residuals of the fourcomponent model (Fig. 2a); however, this peak was highlighted in the plot of $\Delta R_{4-5 C}$ (Fig. 2b), and was consequently absent from the mean residuals of Tamarack leachates in the five-component PARAFAC model (Fig. 2c). A proteinpolyphenol-like peak persisted in the mean residuals of the overwintered silver maple leachates, requiring nine PARAFAC components for adequate removal (e.g., see Fig. 2b, lower panel, and Figs. 2d). No fluorophore-like fluorescence remained in the overall mean or species-specific 
residuals after the ninth component was removed. Although some fluorescence was evident along the scatter line in the mean residuals (Fig. 2a, lower panel), this was not considered chemically meaningful since this region is frequently associated with noise caused by incomplete scatter removal. $^{12}$

In PARAFAC models of the LR data set, $\Delta R$ plots evidenced changes that were primarily associated with a single peak, but also described complex refinements to the loadings of several components (Fig. 3). This is likely because peaks were more evenly distributed among the samples and the components remaining in the residuals explained a lesser amount of total variance, so that they were closer to the noise level (Fig. SI6). This effect was especially pronounced during the transition between the four- and five-component PARAFAC models, where the splitting of a broad humic-like peak with $E x / E m=235$ / $425 \mathrm{~nm}$ into two humic-like peaks $(\mathrm{Ex} / \mathrm{Em}=235 / 430$,
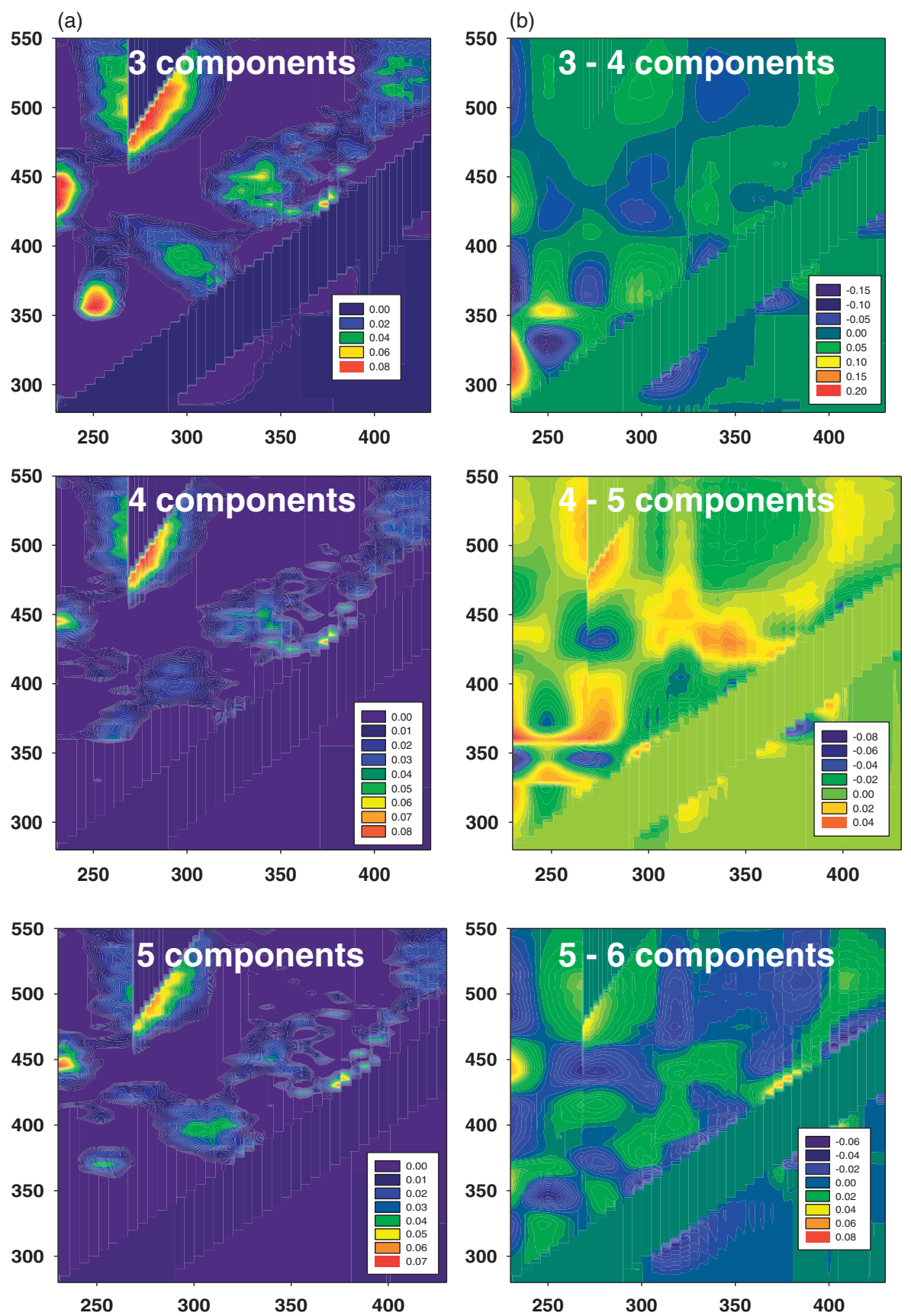

Figure 3. (a) Overall mean residuals, and (b) $\triangle R$ plots for PARAFAC models of the LR data set with three to five components. Excitation and emission wavelengths are shown along the $x$ - and $y$-axes, respectively. 
$235 / 410 \mathrm{~nm}$; Figs. SII and SI2) primarily affected the loadings of apparently disconnected protein- and humic-like fluorescence $\quad(\mathrm{Ex} / \mathrm{Em}=<230(275) / 360, \quad$ approximately $275 / 510$, and $345 / 440 \mathrm{~nm}$; Fig. 3b). The majority of the chemically meaningful fluorescence was removed in the six-component model of the LR data set (lower panels of Fig. 3), and fluorescence in the $\Delta R_{6-7 c}$ plot was primarily associated with small peaks near the diagonal of the removed scatter (data not shown). Thus, the direct inspection of residuals suggests that the LR data set is adequately described by six PARAFAC components.

\section{Pattern Detection in Residuals}

Using the SOM, the residuals of the three-component PARAFAC models were organized into groups with similar residual EEMs. Thus, any patterns that are evident in the EEMs of the SOM map nodes ("neuron EEMs") reflect the dominant patterns in the residuals from the MS and LR data sets (Fig. $4 a$ and $b$ ). Neuron EEMs are shown for the nodes in the map corners and centers to demonstrate the range of patterns, since neurons on opposite edges-corners of the map differ the greatest (SOM nodes are numbered from top to bottom, and left to right). Thus, neuron EEMs from map corners demonstrate the range of fluorescence characteristics that are most useful for distinguishing samples based on the patterns that remain in the residuals, while the EEMs from the map center indicate more average features.

The neuron EEMs from the residuals of the threecomponent model of the MS data set contained three distinct protein-like peaks (neurons N3, N33, and N40), one humic-like peak (NI), and a large band of humic-fulvic-like fluorescence (N8; Fig. 4a). These EEMs were primarily associated with the same peaks and tree species as in $\Delta \mathrm{R}$ plots (Fig. 2b). For example, the mean residuals from the Tamarack leachates (STL) contained peaks near $\mathrm{Ex} / \mathrm{Em}=235 / 3 / 5,255 / 380$, and $3 / 5 / 430 \mathrm{~nm}$ (upper panel, Fig. 2c). Consequently, Tamarack leachates were primarily associated with map node/neuron three in the converged SOM (dark blue circle, Fig. 5a) and its corresponding neuron EEM contained fluorescence in these regions (N3, Fig. 4a). Further, the neuron EEMs for node one (upper lefthand corner) and node eight (lower left-hand corner) contained subsets of these peaks, so that the Tamarack leachates were placed in the first column of the SOM map. The EEM of neuron 40 (Fig. 4a) was dominated by the proteinlike peak that remained in the residuals of the eight-component model for the overwintered silver maple leachates (a)
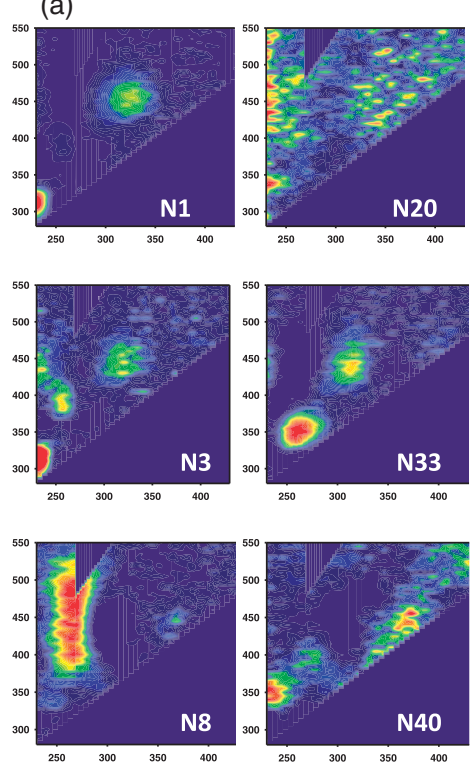

(b)
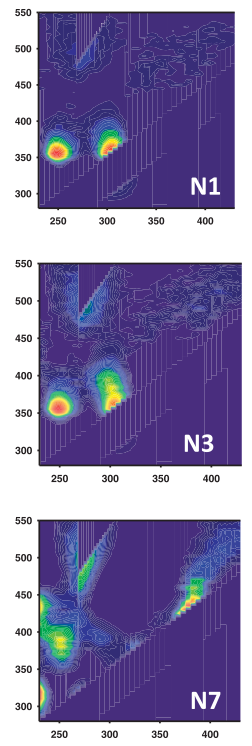
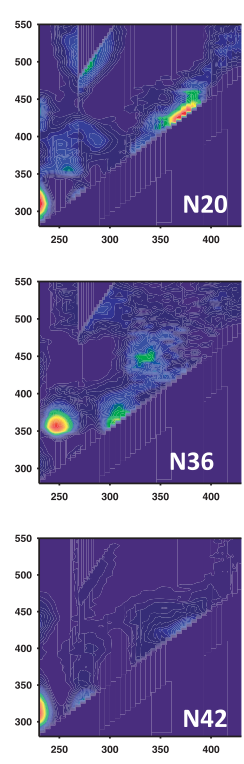

(c)
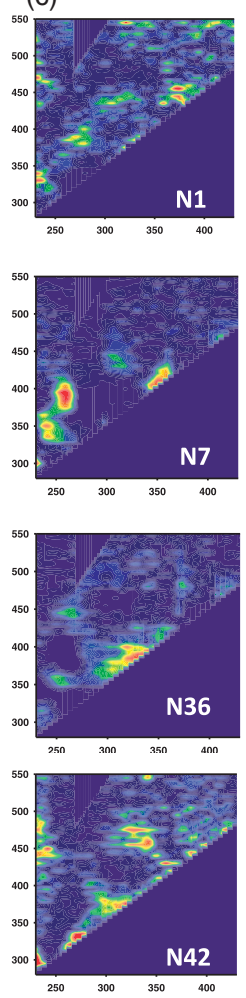

(d)
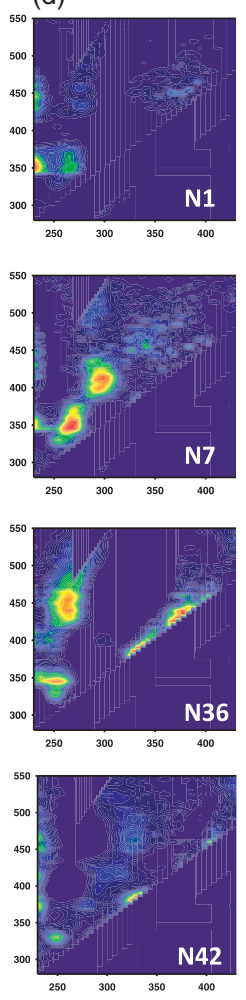

Figure 4. Neuron EEMs from SOMs of residuals from three-component PARAFAC models of the (a) MS and (b) LN data sets, and for the (c) nine-component model of the MS data set and (d) six-component model of the LN data set. EEMs are designated by their associated map neuron $(\mathrm{N})$, where neurons are numbered first from top to bottom, and from left to right. Excitation and emission wavelengths are shown along the $x$ - and $y$-axes, respectively. 
(a)

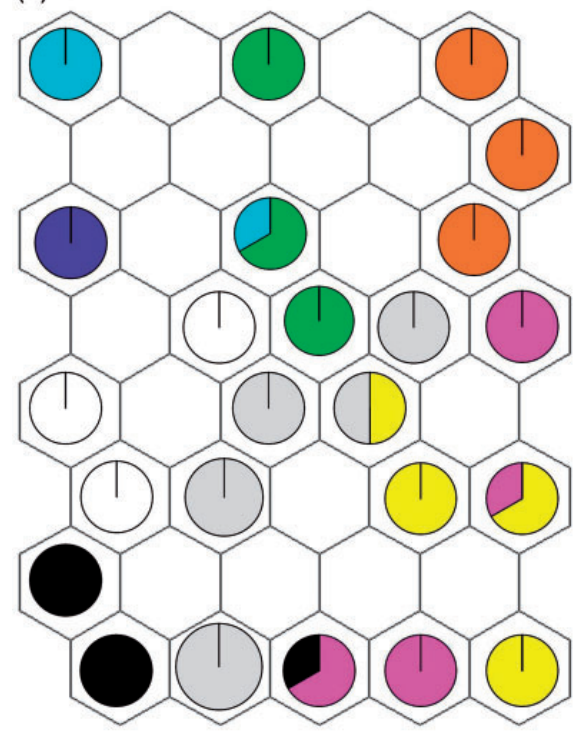

(b)

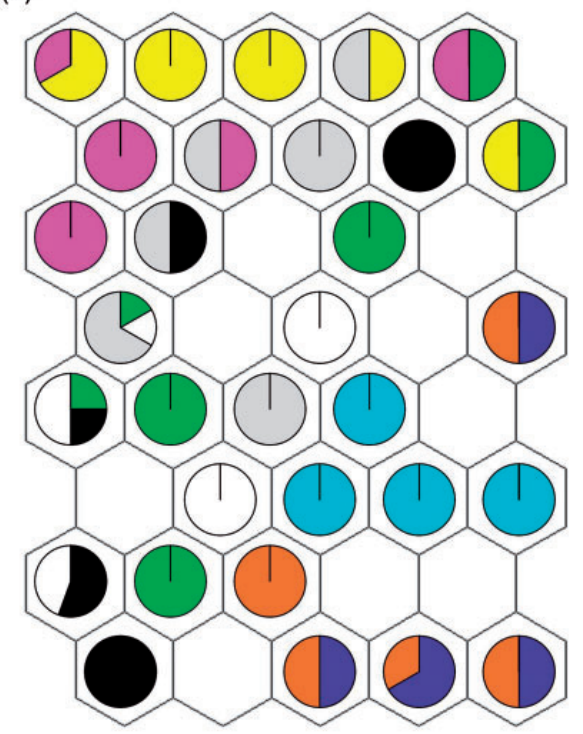

Figure 5. Distribution of DOM by tree species-headwater on SOMs based on PARAFAC models of the MS data set for (a) residuals from the three-component model, and (b) component loadings from the three-component model. Circles in map nodes are colored according to species-headwater (๑ Red maple; Overwintered silver maple; $\bullet$ Senescent silver maple; $\bullet$ Spruce; $\bullet$ Tamarack; $\bullet$ Canarygrass; • Wetland; $\bigcirc$ Deciduous headwater; - Coniferous headwater). Neurons are numbered first from top to bottom, and from left to right.

(Fig. 2d). Fittingly, residual EEMs from overwintered silver maple leachates were most associated with neuron 40 , in the lower right-hand corner of the SOM (Fig. 5a). It is clear that important information remained in the residuals of the three-component model, particularly given that the organization of EEMs by tree species was better in the SOM of the residuals from the three-component PARAFAC model than in the SOM of the component loadings (Fig. 5a and b).

Neuron EEMs for the SOM of residuals from the ninecomponent PARAFAC model of the MS data set were dominated by noise and fluorescence near the area where scatter was removed (Fig. 4c), demonstrating that no chemically meaningful fluorescence could be isolated. Coupled with the $\Delta R$ plots (Fig. $2 b$ ) and plots of mean residuals by tree species (Fig. 2c and d), this suggests that systematic fluorescence in the MS data set is completely described using a nine-component PARAFAC model.

Patterns in the neuron EEMs of the residuals from the three-component PARAFAC model of the LR data set also resembled the peaks evident in $\Delta R$ plots and plots of mean residuals (Figs. 3 and $4 \mathrm{~b}$ ). These patterns were removed by the addition of PARAFAC components, but a few peak-like features remained in the residuals of the six-component model (Fig. 4d). However, several features were also evident along the edge of the region that was removed as scatter, which were the dominant features removed by the seven-component PARAFAC model (data not shown). Consequently, to avoid the potential inclusion of noise the PARAFAC model of the LR data set could not be extended beyond six components.

\section{Assessing Overfitting and Comparing Models}

If the PARAFAC model was overfitted, then one or more components would contain significant noise. Such superfluous components would not contribute to the differentiation of samples because they would be distributed quasievenly. Hence, the clustering of EEMs by tree species and corresponding distributions of component loadings (Fmax) on the converged SOM of the nine-component PARAFAC model were used to assess noise levels and the likelihood of overfitting (Fig. 6). Relatively high loadings on $\mathrm{Cl}, \mathrm{C} 4, \mathrm{C} 6$, and $\mathrm{C} 7$ served to distinguish headwaters and leachates dominated by fulvic- and humic-like fluorescence (Fig. I) from the leachates of needles, red maple, and sugar maple, which were dominated by protein/polyphenollike $\mathrm{C} 2$ and $\mathrm{C} 5$, microbial humic-like $\mathrm{C} 3$, and humic-like $\mathrm{C}$. Loadings on these eight components also distinguished tree species and headwaters within these humic- and protein/ polyphenol-dominated groups. For example, $\mathrm{Cl}$ and $\mathrm{C} 7$ were higher for the headwaters, while over-wintered silver maple had relatively high loadings on $\mathrm{C} 4$ and $\mathrm{C} 6$, but low loadings on C7. Relatively low levels of $C 2$ and high C5 distinguished senescent sugar maple from tamarack and white spruce, while levels of humic-like $\mathrm{C} 9$ were much higher in spruce compared to tamarack. High levels of microbial humic-like $\mathrm{C} 3$ characterized the leachates of senescent red maple leaves. Loadings on protein-polyphenollike C8 were very high for overwintered silver maple, medial for senescent sugar maple, and lower for all other samples. Thus, each component served to distinguish 
samples, was not highly correlated with other components, and was not randomly distributed. Thus, it is unlikely that the nine-component PARAFAC model was overfitted.

The larger size fractions of all samples were clustered in the center of the map owing to the relatively large proportion of humic-like fluorescence and less DOM, making them less distinct by tree species or headwater (sizes shown in Cuss and Guéguen ${ }^{21}$ ). For example, protein-polyphenollike C2, C5, and C8 were virtually absent from the larger size fractions of the humic-fulvic-dominated samples in the center of the map, while $\mathrm{Cl}$ and $\mathrm{C} 4$ were present at medial levels. Differences in the concentration of DOM in different size fractions thus limited the extent to which samples could be distinguished by species/headwater.

To assess the value of using residuals analysis to extract all pertinent spectral information, the converged SOM and corresponding component distributions were compared for the PARAFAC model of the MS data set validated by split-half analysis (three components; Fig. 5b) and by advanced residuals analysis (nine components; Fig. 6b). Although the humic-fulvic-dominated and protein/polyphenoldominated samples were mostly clustered in opposite corners of the map for the three-component model (i.e., upper left versus lower right; Fig. 5b), they were much easier to distinguish in the nine-component model (Fig. 6b). Differences in differentiability between the two models were quantified by comparing the proportions of both size-fractionated and whole-sample EEMs that were correctly classified by the $k$-nearest neighbor algorithm. The three-component model facilitated correct classification of $56.0 \pm 0.8$ and $51.7 \pm 1.4 \%$ of the size-fractionated and whole-sample EEMs, respectively, while the ninecomponent model correctly classified $75.2 \pm 0.9$ and $92.9 \pm 0.0 \%$, respectively. This large difference is likely because the three-component model was limited to explaining differences using only components that were present in the majority of samples. Obviously, this can place serious limitations on differentiation in data sets that contain distinct fluorophores. Finally, the extent of this limitation is underscored by comparing the converged SOM for the three-component model loadings (Fig. 5b) to that of the three-component model residuals (Fig. 5a), from which it is clear that the residuals of the three-component PARAFAC model describe the sample-specific nature of the samples better than the PARAFAC model itself. This finding stresses the fact that useful information can be lost by relying upon split-half analysis for validating the number of PARAFAC components in models of DOM fluorescence, and emphasizes the importance of using other diagnostics such as advanced residuals analysis to enhance the number of components.

Extrapolating the number of PARAFAC components beyond what is validated by split-half analysis should be undertaken with caution. This study has demonstrated how the rigorous analysis of PARAFAC models and their residuals can be used to develop a fine-grained description of DOM fluorescence in sets of EEMs with disparate fluorescence properties. This careful analysis relied upon five (a)
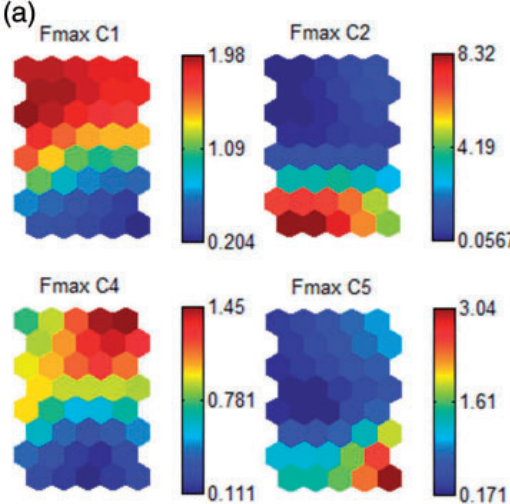

$\mathrm{Fmax} C 7$

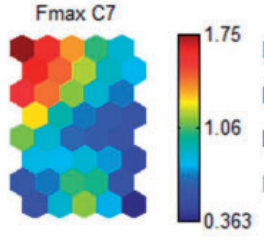

$\mathrm{F} \max \mathrm{C} 2$

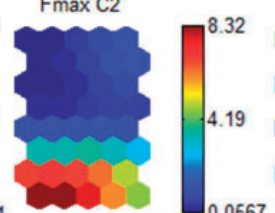

Fmax $\mathrm{C8}$
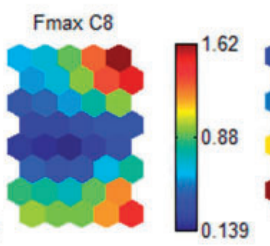
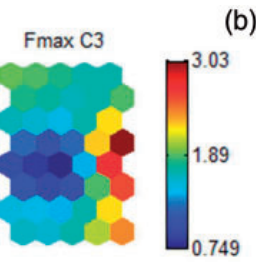

$\mathrm{F} \max \mathrm{C6}$

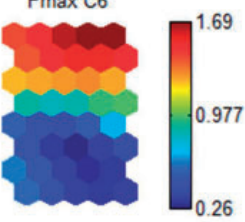

$\mathrm{Fmax} C 9$
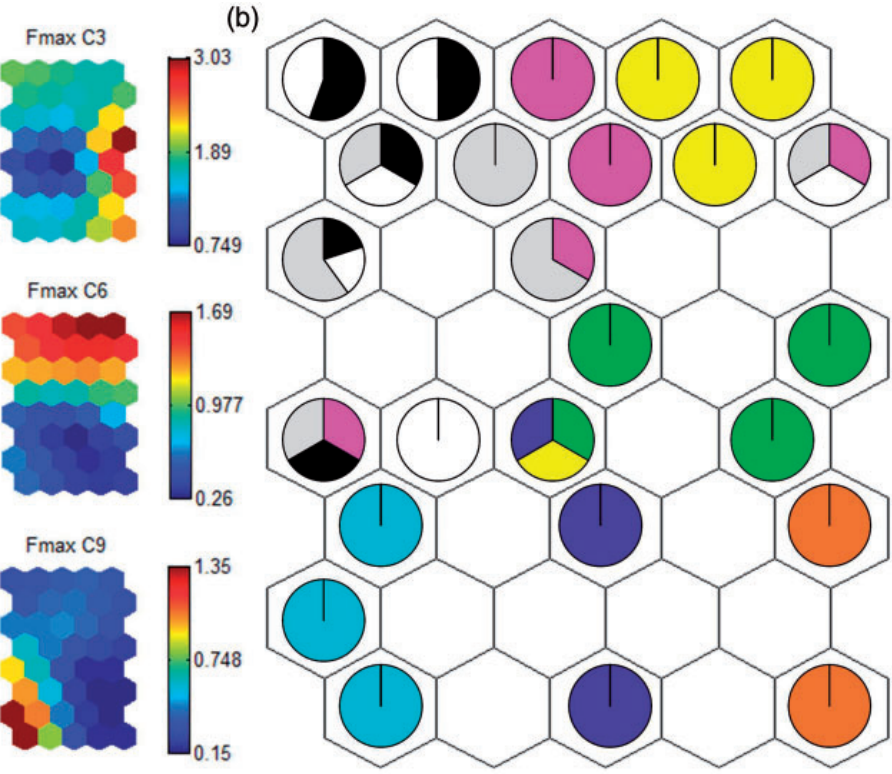

Figure 6. Distributions of (a) component loadings, and (b) sample EEMs on the SOM of the nine-component PARAFAC model of the MS data set. Circles in map nodes are colored according to tree species/headwater ( Red maple; Overwintered silver maple; $\bullet$ Senescent silver maple; $\bullet$ Spruce; • Tamarack; • Canarygrass; • Wetland; $\bigcirc$ Deciduous headwater; • Coniferous headwater). Neurons are numbered first from top to bottom, and from left to right. 


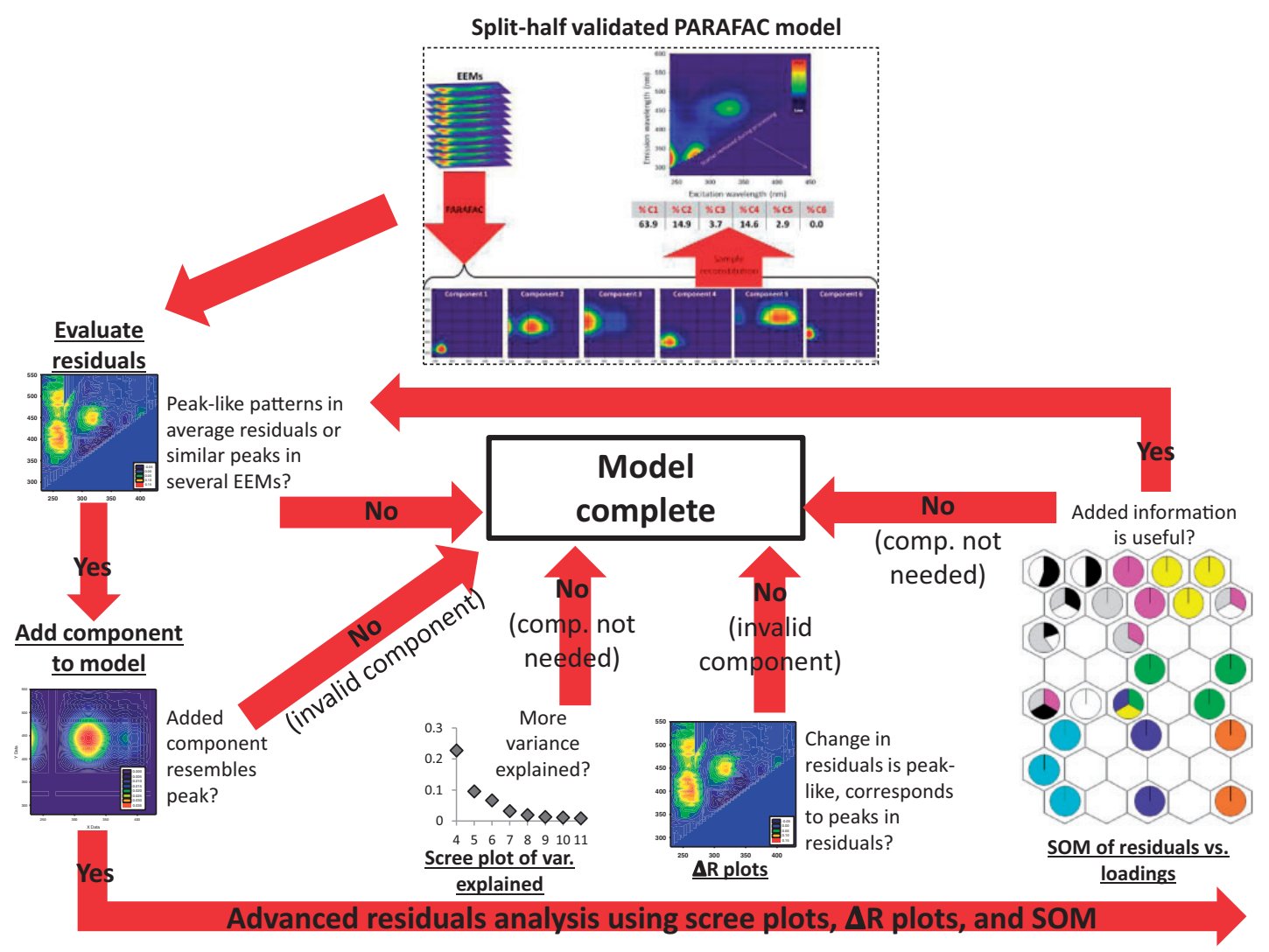

Figure 7. Schematic of steps used to assess the value and validity of adding components to the split-half validated PARAFAC model.

major indicators to extend the PARAFAC model: (I) scree plot evidencing increases in variance explained by adding components; (2) confirmation that the spectra of additional components were fluorescent peaks and not dominated by noise; (3) $\Delta R$ plots to ensure that the change in residuals reflected a fluorescence peak when a component was added; (4) examination of average residuals plots for collections of samples from distinct species, and comparing components added to the impact on residuals (i.e., $\Delta R$ plots); and (5) methods for unsupervised pattern recognition in residuals and PARAFAC component loadings (i.e., SOM) to assess the amount of information/fluorescence peaks that remain in residuals (Fig. 7). Importantly, the amount of useful information contained in residuals could be compared with that in the PARAFAC model using SOM and $k$-means clustering owing to the inclusion of several sample types with disparate fluorescence properties (i.e., leaf leachates).

\section{Conclusion}

Parallel factor analysis is the dominant method for decomposing the complex fluorescence EEM spectra of dissolved organic matter because it is relatively simple to apply, well developed, and applies a standardized approach for extracting chemically meaningful signals. Accordingly, splithalf analysis is the primary method for validating PARAFAC models because it provides robust protection from overfitting. However, this study has demonstrated that validation may miss important information in data sets that contain DOM with disparate fluorescence properties. It has also been demonstrated that such information loss may inadequately represent the distinctiveness of different DOM, which is one of the principal applications for both PARAFAC and DOM research generally. Hence, methods that supplement split-half analysis are desirable. However, caution is advised when extending PARAFAC models beyond the number of components suggested by split-half analysis. This study has demonstrated several methods by which additional components can be evaluated for their value and validity. We hope that this study will assist researchers in studying the biogeochemical cycling of DOM by facilitating the development of high resolution PARAFAC models.

\section{Acknowledgments}

The authors wish to thank A McDonough for tree species identification, and $\mathrm{S}$ Watmough and $\mathrm{K}$ Pinder for assistance with the collection of headwater samples. The helpful comments of two anonymous reviewers are greatly appreciated. 


\section{Funding}

This work was funded in part by the Canada Research Chairs program, Natural Sciences and Engineering Research Council grants (CG). CWC gratefully acknowledges the financial support accorded by the Alexander Graham Bell Canada Graduate Scholarship (NSERC).

\section{Conflict of Interest}

The authors report there are no conflicts of interest.

\section{Supplemental Material}

All supplemental material mentioned in the text, including: a description of the quality testing parameters and parameter settings for self-organizing maps and k-nearest neighbor algorithms, 16 figures with full spectra for all PARAFAC model components, and scree plots for models of the two data sets with increasing numbers of PARAFAC components, is available in the online version of the journal, is available at http://asp.sagepub.com/ supplemental.

\section{References}

I. T.N. Wiegner, L.A. Kaplan, J.D. Newbold, P.H. Ostrom. "Contribution of Dissolved Organic C to Stream Metabolism: A Mesocosm Study Using I3C-Enriched Tree Tissue Leachate". J. N. Am. Benthol. Soc. 2005. 24(I): 48-67.

2. R.G. Zepp, D.J. Erickson III, N.D. Paul, B. Sulzberger. "Interactive Effects of Solar UV Radiation and Climate Change on Biogeochemical Cycling”. Photochem. Photobiol. Sci. 2007. 6: 286-300.

3. J.B. Fellman, E. Hood, R.G.M. Spencer. "Fluorescence Spectroscopy Opens New Windows into Dissolved Organic Matter Dynamics in Freshwater Ecosystems: A Review". Limnol. Oceanogr. 2010. 55(6): 2452-2462.

4. C. Guéguen, J. Dominik. "Partitioning of Trace Metals Between Particulate, Colloidal and Truly Dissolved Fractions in a Polluted River: The Upper Vistula River (Poland)". Appl. Geochem. 2003. 18(3): 457-470.

5. P.F.M. Nogueira, M.G.G. Melão, A.T. Lombardi, M.M. Nogueira. "Natural DOM Affects Copper Speciation and Bioavailability to Bacteria and Ciliate". Arch. Environ. Contam. Toxicol. 2009. 57(2): 274-28I.

6. C.W. Cuss, C. Guéguen. "Impacts of Microbial Activity on the Optical and Copper-Binding Properties of Leaf-Litter Leachate". Front. Microbiol. 2012. 3: 166.

7. T.E. Kraus, C.A. Anderson, K. Morgenstern, B.D. Downing, B.A. Pellerin, B.A. Bergamaschi. "Determining Sources of Dissolved Organic Carbon Disinfection Byproduct Precursors to the Mackenzie River, Oregon". J. Environ. Qual. 2010. 39(6): 2100-2II2.

8. K.M.H. Beggs, R.S. Summers. "Character and Chlorine Reactivity of Dissolved Organic Matter from a Mountain Pine Beetle Impacted Watershed". Environ. Sci. Technol. $201 \mathrm{I}$. 45(I3): 57I7-5724.

9. J. Hur, H.V.-M. Nguyen, B.-M. Lee. "Influence of Upstream Land Use on Dissolved Organic Matter and Trihalomethane Formation Potential in Watersheds for Two Different Seasons”. Environ. Sci. Pollut. Res. 2014. 2I(I2): 7489-7500.
10. P.G. Coble. "Characterization of Marine and Terrestrial DOM in Seawater Using Excitation-Emission Matrix Spectroscopy". Mar. Chem. 1996. 5I(4): 325-346.

II. C.A. Stedmon, R. Bro. "Characterizing Dissolved Organic Matter Fluorescence with Parallel Factor Analysis: A Tutorial”. Limnol. Oceanogr: Methods. 2008. 6: 572-579.

12. K.R. Murphy, C.A. Stedmon, D. Graeber, R. Bro. "Fluorescence Spectroscopy and Multi-Way Techniques. PARAFAC”. Anal. Methods. 2013. 5(23): 6557-6566.

13. G. Aiken. "Fluorescence and Dissolved Organic Matter: A Chemist's Perspective”. In: P. Coble, J. Lead, A. Baker, D. Reynolds, R.G.M. Spencer (eds) Aquatic Organic Matter Fluorescence. New York, NY: Cambridge University Press, 20I4, Chap. 2, Pp.35-74.

I4. C.M. Sharpless, N.V. Blough. "The Importance of ChargeTransfer Interactions in Determining Chromophoric Dissolved Organic Matter (CDOM) Optical and Photochemical Properties”. Environ. Sci.: Processes Impacts. 20I4. 16(4): 654-67I.

I5. C.A. Stedmon, S. Markager. "Resolving the Variability in Dissolved Organic Matter Fluorescence in a Temperate Estuary and Its Catchment Using PARAFAC Analysis". Limnol. Oceanogr. 2005. 50(2): 686-697.

16. C.W. Cuss, C. Guéguen. "Distinguishing Dissolved Organic Matter at Its Origin: Size and Optical Properties of LeafLitter Leachates". Chemosphere. 20I3. 92(II): |483-I489.

17. C.A. Stedmon, S. Markager, R. Bro. "Tracing Dissolved Organic Matter in Aquatic Environments Using a New Approach to Fluorescence Spectroscopy”. Mar. Chem. 2003. 82(3-4): 239-254.

18. C.A. Stedmon, S. Markager. "Tracing the Production and Degradation of Autochthonous Fractions of Dissolved Organic Matter by Fluorescence Analysis". Limnol. Oceanogr. 2005. 50(5): |4I5-| 426.

19. M. Chen, R. Jaffé. "Photo- and Bio-Reactivity Patterns of Dissolved Organic Matter from Biomass and Soil Leachates and Surface Waters in a Subtropical Wetland". Wat. Res. 2014. 61: $181-190$.

20. C.W. Cuss, C. Guéguen. "Determination of the Relative Molecular Weights of Fluorescent Components in Dissolved Organic Matter Using Asymmetrical Flow Field-Flow Fractionation and Parallel Factor Analysis". Anal. Chim. Acta. 2012. 733: 98-102.

2I. C.W. Cuss, C. Guéguen. "Relationships Between Molecular Weight and Fluorescence Properties for Size-Fractionated Dissolved Organic Matter from Fresh and Aged Sources". Wat. Res. 20I5. 68: 487-497.

22. C. Romera-Castillo, M. Chen, Y. Yamashita, R. Jaffé. "Fluorescence Characteristics of Dissolved Organic Matter: Implications for a Molecular Assembly Based Structure?" Wat. Res. 2014. 55: 40-5I.

23. S. Singh, S. Inamdar, D. Scott. "Comparison of Two PARAFAC Models of Dissolved Organic Matter Fluorescence for a MidAtlantic Forested Watershed in the USA". J. Ecosyst. 2013. 2013: 532424.

24. R.A. Harshman. "'How Can I Know If It's 'Real?' A Catalog of Diagnostics for Use with Three-Mode Factor Analysis and Multidimensional Scaling”. In: H.G. Law, C.W. Snyder Jr., J.A. Hattie, R.P. McDonald (eds) Research Methods for Multimode Data Analysis. New York, NY: Praeger, 1984, pp.566-591. 
25. T. Kohonen. Self-Organizing Maps (3e). Dordrecht, Germany: Springer-Verlag, 200I.

26. C. Guéguen, C.W. Cuss. "Characterization of Aquatic Dissolved Organic Matter by Asymmetrical Flow Field-Flow Fractionation Coupled to UV-Visible Diode Array and Excitation Emission Matrix Fluorescence". J. Chromatogr. A. 20II. 1218(27): 4188-4198.

27. J.R. Lakowicz. Principles of Fluorescence Spectroscopy, 3rd ed. New York: Plenum, 2006.

28. A.J. Lawaetz, C.A. Stedmon. "Fluorescence Intensity Calibration Using the Raman Scatter Peak of Water". Appl. Spectrosc. 2009. 63(8): 936-940.

29. M. Cottrell, P. Gaubert, C. Eloy, D. François, G. Hallaux, J. Lacaille, M. Verleysen. "Fault Prediction in Aircraft Engines Using Self-Organizing Maps". Proceedings of the 7th International Workshop on Advances in Self-Organizing Maps. 2009. doi: 10.1007/978-3-642-02397-2_5.

30. M. Bieroza, A. Baker, J. Bridgeman. "Exploratory Analysis of Excitation-Emission Matrix Fluorescence Spectra with SelfOrganizing Maps as a Basis for Determination of Organic Matter Removal Efficiency at Water Treatment Works". J. Geophys. Res. 2009. II4: G00F07.

3I. M. Bieroza, A. Baker, J. Bridgeman. "Exploratory Analysis of Excitation-Emission Matrix Fluorescence Spectra with SelfOrganizing Maps-A Tutorial". Edu. Chem. Eng. 2012. 7(I): e22-e3l.

32. E.M. Carstea, A. Baker, M. Bieroza, D. Reynolds. "Continuous Fluorescence Excitation-Emission Matrix Monitoring of River Organic Matter”. Wat. Res. 2010. 44(18): 5356-5366.

33. E. Ejarque-Gonzalez, A. Butturini. "Self-Organising Maps and Correlation Analysis as a Tool to Explore Patterns in Excitation-Emission Matrix Data Sets and to Discriminate Dissolved Organic Matter Fluorescence Components". PLOS One. 2014. 9(6): e99618.
34. C.W. Cuss, Y.X. Shi, S.M. McConnell, C. Guéguen. "Changes in the Fluorescence Composition of Multiple DOM Sources over $\mathrm{pH}$ Gradients Assessed by Combining Parallel Factor Analysis and Self-Organizing Maps". J. Geophys. Res: Biogeosci. 2014. II9(9): 1850-1860.

35. A.M. Kalteh, P. Hjorth, R. Berndtsson. "Review of the SelfOrganizing Map (SOM) Approach in Water Resources: Analysis, Modelling and Application”. Environ. Model. Soft. 2008. 23(7): 835-845.

36. S.J. Ki, J.-H. Kang, S.W. Lee, Y.S. Lee, K.W. Cho, K.-G. An, J.H. Kim. "Advanced Assessment and Design of Stormwater Monitoring Programs Using a Self-Organizing Map: Characterization of Trace Metal Concentration Profiles in Stormwater Runoff”. Wat. Res. 20II. 45(I4): 4I83-4I97.

37. N. Roig, M. Nadal, J. Sierra, A. Ginebreda, M. Schuhmacher, J.L. Domingo. "Novel Approach for Assessing Heavy Metal Pollution and Ecotoxicological Status of Rivers by Means of Passive Sampling Methods". Environ. Int. 20II. 37(4): 67I-677.

38. S. Tsakovski, B. Kudlak, V. Simeonov, L. Wolska, J. Namiesnik J. "Ecotoxicity and Chemical Sediment Data Classification by the Use of Self-Organising Maps". Anal. Chim. Acta. 2009. 63I(2): 142-152.

39. V. Pawlowsky-Glahn, J.J. Egozcue. "Compositional Data and Their Analysis: An Introduction”. London Geological Society Special Publications. 2006. 264: I-I0.

40. R. Bro. "PARAFAC. Tutorial and Applications". Chemometr. Intell. Lab. 1997. 38(2): |49-I7I.

4I. R. Bro, H.A.L. Kiers. "A New Efficient Method for Determining the Number of Components in PARAFAC Models". J. Chemom. 2003. 17(5): 274-286.

42. P.M. Kroonenberg. Applied Multiway Data Analysis. Hoboken, NJ: John Wiley \& Sons, 2008. 